\title{
Der Gedanke des Eifers Jahwes im Alten Testament.
}

\author{
Von Privatdozent Lic. Dr. Friedrich Küchler in Berlin.
}

Wenn wir dem Gedanken vom Eifer Jahwes, der im AT eine gewisse Rolle spielt, nachgehen wollen, so müssen wir uns zunächst mit seinem sprachlichen Ausdruck vertraut machen. Es handelt sich dabei lediglich um das Substantivum קִָָּ und die davon abgeleiteten Verbalformen und Adjektiva. Letztere gehören sämtlich dem Steigerungsstamme, die ersteren diesem und dem Kausativstamme an.

Eine einwandfreie Etymologie für das Wort הִֶ? gibt es nicht. مạtlich unbedenklich ist zwar seine Zusammenstellung mit aram. und den davon abgeleiteten Verbalformen, mögen diese Wörter nun mit a oder mit $\zeta$ geschrieben werden (das 'Ajin geht jedenfalls auf eine bekanntlich häufige Angleichung der Konsonanten eines Stammes untereinander, in unserem Falle Angleichung an das emphat. 0 , zurück). Aber sie scheitert daran, daß der ganze Stamm im Aram. aus dem AssyrischBabylonischen entlehnt zu sein und auf das assyrische Wort $u k n \bar{u}$ zurückzugehen scheint, das den Lapis lazuli und dann die blaue Farbe dieses Steines, sowie weiter jede blaue Farbe bezeichnet (JENSEN). Auf diese Bedeutung scheinen mir auch, soweit ich es zu beurteilen vermag, alle in Betracht kommenden Formen von einem arabischen Stamme قَّick zurückzugehen, indem sie sich nicht, wie die Lexica angeben, auf eine rote, sondern auf eine helle oder dunkle blaue Färbung beziehen werden; ${ }^{x}$ auch das griechische kuáveos wird letztlich von dem assyr. $u k n u$ abzuleiten sein. - $\mathrm{Da}$ mit einer solchen Etymologie der Erklärung des hebräischen קניאה und seiner Derivate nicht gedient ist, leuchtet ein. Wir müssen also auf eine Etymologie, die sich auch sonst nicht immer als eine

× Z. B. I. "die Milch wạsșern“ = „blau machen“; "gefärbt sein" (vom Barte), doch wohl blauschwarz, nicht rot. II. "den Bart schwarz färben“. I. u. IV. "töten“ = „blau machen“, vgl. „bleu, mourant".

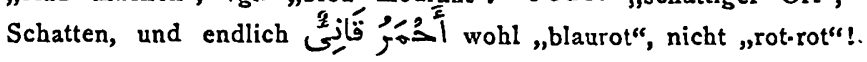


sichere Führerin bewährt, verzichten und den Inhalt der in Betracht kommenden Wörter aus dem Sprachgebrauch des Hebräischen selbst, zunächst natürlich auṣ dem profanen, bestimmen.

Das Substantivum begegnet uns nun in mancherlei Bedeutungen, und mit ihm natürlich auch seine Derivate. Das ursprüngliche Gebiet des Stammes pap scheint mir das der Liebe der Geschlechter und speziell der Ehe zu sein.' Es ist mir wenigstens wahrscheinlicher, daß die Wurzel ursprünglich auf einen ganz bestimmten, eng umgrenzten Ausschnitt des Lebens Anwendung fand, als daß sie von vornherein mit einer ganz allgemeinen Bedeutung, wie etwa "Leidenschaft", behaftet war und deswegen auf alle möglichen Yerhältnisse angewandt werden konnte, wie DUHM das nach einer Bemerkung zu Jes 96 anzunehmen scheint. Es fehlt denn auch nicht an Anhaltspunkten für meine eben geäußerte Meinung. Die sehr merkwürdige und gewiß trotz ihrer späten Überlieferung - sie ist Bestandteil der priesterlichen Schrift - uralte הצובָ Num 5 berichtet davon,

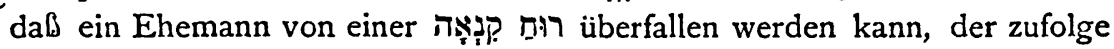
er sein Weib der ehelichen Untreue bezichtigt; die תר gibt nun das magische Verfahren an, durch das festgestellt werden soll, ob der Verdacht des Gatten berechtigt oder grundlos ist. - Die Sprüche, die sich mit dem Thema des Einbruches in fremde Ehe ziemlich ausführlich beschäftigen, nennen als eine seiner schlimmsten Folgen die Țị des hintergangenen Gatten (6 34$)$, die einen unauslöschlichen, aller Schonung und Vergebung abgeneigten Zorn und $\mathrm{Ha} B$ hervorruft (27 4). Hier bezeichnet also pִ die der Liebe von Mann und Weib anhaftende, mit ihr aufs engste verbundenę Sinnesart, die jedes nähere Verhältnis der geliebten Person zu Dritten unerträglich findet; so kommt es, daß im Hohenlied

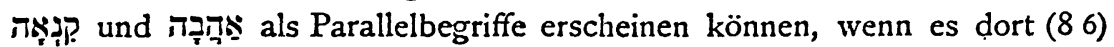

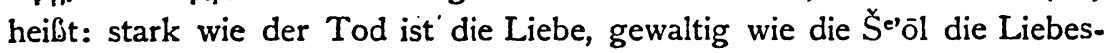
leidenschaft; ihr Brand ist Feuersbrand, eine Jahweflamme.

Kann es so nicht zweifelhaft sein, daß in diesen Fällen der Stamm קנט zur Bezeichnung von Seelenzuständen verwandt wird, die wir mit dem prägnanten Ausdruck Eifersucht $z u$ benennen pflegen, so ist das Gebiet seiner Anwendbarkeit doch erheblich weiter. So hören wir von Rahel (Gen 30 1), daß sie wegen des Umstandes, daß ihrer Schwester Lea Kinder beschieden waren, ihr selbst aber nicht, auf ihre Schwester und Mitgattin eine 
unverständigen und frevelhaften Forderungen plagte. Die Bedeutung des Wortes Nop ist hier noch ziemlich nahe mit der Eifersucht im engeren Sinne verwandt; sie geht aber doch schon über zur Bedeutung des Neidens oder Mißgünstigseins. In dieser findet die Wurzel ebenfalls

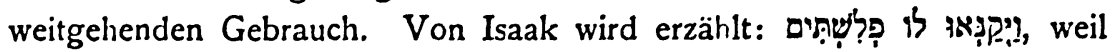
sein Besitz überaus groß wurde (Gen 26 14), und den Josef traf die seiner Brüder, als sie bemerkten, daß er seinen Sinn zu Höherem erhebe und wohl auch bestimmt sei als sie (Gen $37 \mathrm{Ir}$ ). Dab man den Gottlosen nicht um sein Glück beneiden soll (durch wiederholte Mahnung des Spruchbuches (3 31 23 1). Nach Hes 31 I9 beneideten alle Bäume von Eden die von Gott gepflanzte herrliche Zeder

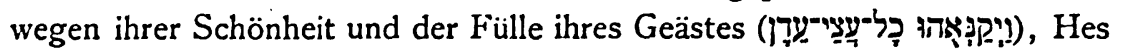
35 I wird der Neid Edoms, in dem er Juda sein Land nicht gönnte, als

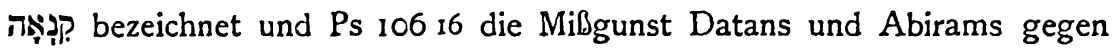
Mose und Aaron wegen ihrer bevorzugten Stellung ebenfalls durch das Verbum p? pausgedrückt. Endlich erwartet ein später Schriftsteller im

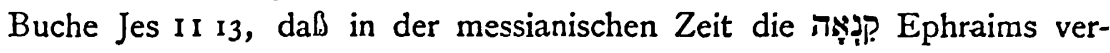
schwinden und die Dränger Judas ausgetilgt werden, womit entgegen der Meinung eines noch späteren Glossators nur gesagt sein kann, da@ in der Endzeit Ephraim unbeneidet und Juda unbefeindet dastehen und sich beständigen Friedens und Wohlergehens erfreuen werden.

Mit dem Neid nahe verwandt ist das Streben, selbsit das zu erreichen, was man von einem andern erreicht oder wonach man ihn streben sieht; auch diese Nuance nimmt die Wurzel קנז im Hebräischen an. Es scheint wenigstens, daß man Koh 44 von dem gegenseitigen Wetteifer der Menschen um die Erreichung eines gemeinsamen Zieles verstehen muß, sicherlich ein wertvolles Motiv für einen Menschen, das ihn zur Einsetzung seiner besten Kräfte für die Erfüllung der einmal erkannten Aufgabe anspornen kann; der pessimistische Autor des Buches Kohelet tut es

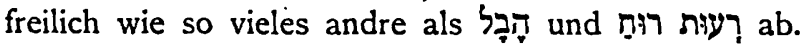

Noch weiter abgeblaßt erscheint endlich die Bedeutung unsres Stammes da, wo er nichts weiter als den unmutigen Affekt und das Inaffektgeraten ganz im allgemeinen, sowie das Handeln im Affekt bezeichnet. Auch diese Bedeutungsnuance ist im AT ziemlich häufig. So steht א. es heißt: erhitze dich nicht über die Bösewichter und gerate nicht in Unmut über denen, die unrecht handeln, oder Prv 2317 und 24 19, wo davor gewarnt wird, sich an den Sündern zu erregen; das solle man vielmehr an der Furcht Jahwes tun. Daß er sich über das Wohlergehen 
der Gottlosen aufgeregt habe, beklagt der Dichter von Ps 73 nachträglich (v. 3), und der langatmige Kompilator von Ps I 19 erwähnt den Schaden, den seine habe (v. 139). Vor dem Affekt überhaupt warnt Prv 14 30, wo die in Gegensatz zu einem geruhigen Herzen gestellt wird; ganz ähnlich beurteilt der Dichter des Hiob das als קִּנִ pezeichnete Abweichen von der stoischen Ruhe des Gemüts ( 52 ) und der Prediger vergibt, wenn er den Zustand der Toten beschreibt, nicht $z \mathfrak{u}$ erwähnen, daß bei ihnen nun Liebe und $\mathrm{HaB}$ und daß sie somit keinerlei Anteil mehr haben an allem, was auf Erden geschieht (96).

Wir kommen nun noch zu den Stellen, in denen die pִָ̧ auf eine dritte Person bezogen erscheint, in deren Interesse sie liegt; diese Person wird durch die Präposition ? eingeführt. So hören wir von Saul (II Sam

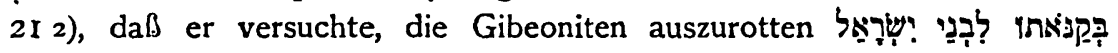

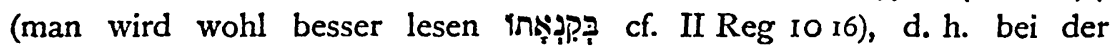
temperamentvollen Wahrnehmung der israelitischen Interessen. Ebenso bezeichnet das Verbum ?ִ perhalten Josuas, als er versuchte Moses angebliche Interessen gegenüber den geisterfüllten Gliedern der Gemeinde wahrzunehmen (Num I I 29).

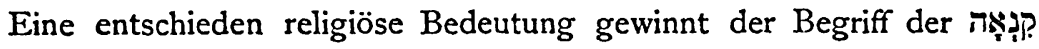
da, wo die Person, in deren Interesse Affekthandlungen unternommen werden, die Gottheit ist. In den drei Fällen, die in dieser Beziehung in Betracht kommen (Pinchas, Elia und Jehu Num 25, I Reg I9 und II Reg I0 16), handelt es sich jedesmal um das Eintreten der genannten Männer für die alleinige Verehrung Jahwes und gegen den Götzendienst, also um die leidenschaftliche Wahrnehmung der Interessen der Gottheit, wenn nicht etwa in diesem Sprachgebrauch erst eine Nachbildung des von der Gottheit selbst aúgesagten werden, ursprünglich ganz allein auf den Götzendienst bezieht. Ähnlich

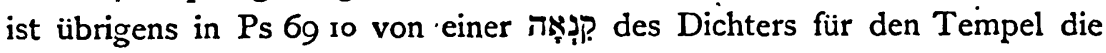
Rede, die so groß war, da@ er sagen konnte, sie habe ihn ganz verzehrt.

II.

Wie steht es nun in den Fällen, wo im AT von einer Ț Jahwes geredet, wo Jahwe als ein gesagt wird, man habe ihn in den Zustand der

Die ältesten Stellen, in denen die Wurzel p pich auf Jahwe angewendet findet, sind Ex 2053414 und Jos 24 19. Weiter kommen als 
vorexilisch noch mehrere Stellen aus dem Deuteronomium und eine aus dem Königsbuche (I Reg 1422) in Betracht. Andere Stellen, in denen der קִ Jahwes gedacht wird, die man oft als vorexilisch auffalit, sind mir bei näherer Untersuchung alle ins Exil oder noch tiefer hinab gerückt.

Daß die drei erstgenannten Stellen vorexilisch seien, ist wohl allgemein anerkannt. Die gehören ja der jahwistischen und der elohistischen Quelle des Hexateuchs an. Nur will es das Unglück, daf gerade die Zusammenhänge, in denen sie stehen, nicht den ungetrübten Strom der alten Quelle erkennen lassen, sondern alle mehr oder weniger stark überarbeitet sind. So sind Ex 20 und Jos 24 sicher durch deuteronomistische - Hände verändert, was man von Ex 34 nicht sagen kann; aber auch der sogenannte jahwistische Dekalog dieses Kapitels hat doch Erweiterungen erfahren, die WELlHAUSEN ,alt“ nennt und KUENEN ins 7. Jahrhundert setzt. Zu diesen Zusätzen gehört nun auch der für uns hier in Betracht kommende Teil von v. 14 nebst dem folgenden. $\mathrm{Da}$ v. 15 nicht dem alten jahwistischen Bestande dieses Abschnittes angehören kann, bedarf keines Nachweises. Er setzt in seiner Terminologie zweifellos die Verkündigung Hoseas voraus, der, soviel wir wissen, zuerst die Verehrung anderer Götter als Hurerei und Ehebruch bezeichnet hat. Es ist mir aber nicht unwahrscheinlich, daß dasselbe auch von v. $14 b$ gilt. Denn erstens paßt zu der Art von „Bundesworten“, die wir nach v. 27 in unserem Abschnitt zu suchen haben, keine Motivierung dieser Gebote, sondern man erwartet nur kurze, knappe, leicht merkbare Sätze, wie WELLHAUSEN sie in seiner Komposition des Hexateuchs aus unserem Abschnitt herausgeschält hat. Muß aus diesem Grunde jedenfalls der weitschweifige v. 15 als der ursprünglichen Konzeption nicht angehörig gestrichen werden, so trifft das auch auf v. $14 \mathrm{~b} z \mathrm{u}$, der für ein kurzes Merkwort schon zu lang erscheint. Zweitens aber setzt auch v. 14 b, wie v. 15, wo das mehr in die Augen springt, die Wirksamkeit des Propheten Hosea voraus, wie dies auch die beiden andern erstgenannten Stellen tun. Die Einführung des

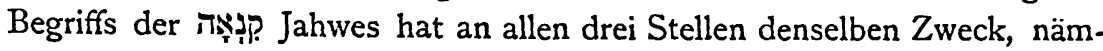
lich den, darauf hinzuweisen, daß mit der Verehrung Jahwes die Verehrung irgendwelcher anderen Götter durch Israel schlechtweg unvereinbar ist; Jahwe will sich eine solche unter keinen Umständen gefallen lassen, er ist ein Gott, der nicht duldet, daß sein Volk sich irgendwie mit anderen Göttern einläßt. Die Idee, die dieser Aussage zugrunde liegt, ist gewiß so alt wie die Jahwereligion in Israel überhaupt. Sie war von Anfang an exklusiv und erhob den Anspruch, die alleinige Religion des Volkes Israel zu sein. Aber die Form des uns hier vorliegenden Ausdrucks für 
diesen Anspruch kann nicht ebenso alt sein; sie wäre für das alte Israel schlechtweg unverständlich gewesen, wenn man nicht annehmen will, daß die Verfasser der uns beschäftigenden Stellen das Wort wạp in einer ganz abgeblaßten Bedeutung auf Jahwe angewendet haben sollten, etwa in dem Sinne von "temperamentvoll". Der Gebrauch eines solchen Wortes in solchem Zusammenhang wäre unendlich matt und schwach und $\mathrm{zu}$ einer solch eindringlichen Mahnung, wie wir sie Ex 20 und 34 lesen, wenig geeignet. Wie ganz anders lebendig aber werden diese Worte, wenn wir für das hebr. אị unser ,eifersüchtig“" in seinem prägnanten Sinne einsetzen dürfen. Da erscheint uns Jahwe als eifersüchtig, wie ein Ehemann auf sein Weib, ein Liebhaber auf die Geliebte eifersüchtig ist. Sowenig diese es ertragen, daß die geliebte Frau sich mit andern Männern abgebe, ja nur mit ihnen tändle, sowenig kann Jahwe es zugeben, daß sein Volk sich mit anderen Göttern einlasse und ihnen Verehrung widme, ja nur nach ihnen schiele. Diese Vorstellung ist aber nur möglich unter der Voraussetzung, daß das Verhältnis Jahwes zu Israel als Ehe aufgefaßt wurde und die Verehrung anderer Götter als Ehebruch. Nun wissen wir aber ganz genau, wo diese Auffassung in der Geschichte des Jahwismus zuerst auftritt: Hosea ist es, der sie eingeführt hat, als ihm die traurigen Erlebnisse seiner eigenen Ehe ein Symbol für das wurden, was Jahwe an seinem Volke erfahren mußte. Damit gewinnen wir einen sicheren terminus a quo für die zeitliche Ansetzung des ersten Vorkommens des Gedankens von der pִִ̣ Jahwes; er ist möglich erst vom letzten Drittel des 8. Jahrhunderts v. Chr. ab.

Man kann es merkwürdig finden, daß bei Hosea selbst der Ausdruck

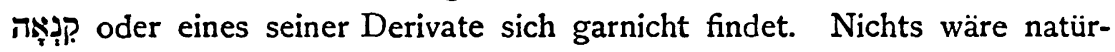
licher, als daß die Ausdrücke bei ihm vorkämen, wo die Sache in so klarer Weise vorliegt, wie dies im 2. Kapitel seines Buches der Fall ist. Aber der Ausdruck fehlt bei ihm so gut, wie bei Jeremia, der, wie bekannt, die Vorstellung von der Ehe Jahwes mit Israel von Hosea übernommen und weitergebildet hat. Es wird wohl so sein, daß diesen Propheten der Ausdruck p̦ nicht zart genug war zur Übertragung auf Jahwe, daß ihm nach ihrer Meinung zu viel Menschliches anhaftete, um auf den Gott, den sie verkündeten und wie sie ihn verstanden, anwendbar zu sein. $\mathrm{Da}$ ähnliche Rücksichten bei Hosea nicht undenkbar wären,

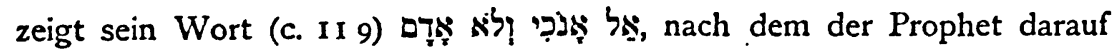
bedacht sein mubte, nicht allzumenschliche Züge in das Bild seines Gottes zu fügen.

Solche feine Rücksichtnahme eignete den Überarbeitern der alten 
Gesctze und Uberlieferungen nicht, sondern sie gebrauchten den Ausdruck ganz unbefangen, und ebenso der Verfasser der historischen Einkleidung des Deuteronomiums. Auch bei ihm ist überall der Zusammenhang mit der Warnung vor dem Götzendienst deutlich; nur tritt bei ihm noch mehr als bei jenen Älteren hervor, daß die ?ִּנִ Jahwes, wenn sie durch Israels Götzendienst wachgerufen wird, Jahwes Zorn zum Ausbruch kommen läbt, der wie ein verzehrendes Feuer das ungetreue Volk vernichtet (Dtn 4 23f. 6 14f. 29 19). Hieran sei gleich angeschlossen, dab auch der Verfasser des deuteronomistischen Rahmens in den Königsbüchern א? demselben Sinne gebraucht, wenn auch nur ein einziges Mal II Reg I4 22. - Endlich halte ich für eben noch vorexilisch das sogenannte Lied des Mose Din 32, das mit deutlicher Anlehnung an Hoseas Gedanken es ganz klar ausspricht, daß der Götzendienst es ist, der Jahwes Volk erweckt.

Bemerkt sei noch, daß schon CALvin (Reliqui quatuor Mosis libri, in formam Harmoniae redacti) in seiner Auslegung des zweiten Gebotes zu den Worten ș bemerkt ,hic vero nobis sub mariti persona proponitur Deus, qui rivalem non patitur", ohne natürlich ahnen zu können, daß die Betrachtung Gottes als maritus seines Volkes sicherlich nicht mosaisch ist, und ferner, daß Steuernagel nach einer Klammer in seinem Kommentar zu Dtn 423 den Zusammenhang der ursprünglichen Vorstellung von der pִ Jahwes mit der Gedankenwelt Hoseas auch schon erkannt hat.

III.

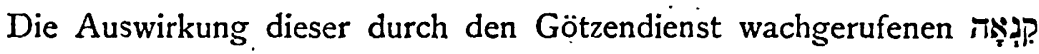
Jahwes glaubte Juda zu erleben in der Vernichtung seines Staatswesens durch Nebukadnezar, in der Deportation des besten Teiles der Nation und der Zerstörung Jerusalems und seines Tempels. Für die Gedanken, mit denen man jene schreckliche Zeit durchlebte, haben wir nur einen Zeugen, Hesekiel. Bei ihm erleben ,wir das Merkwürdige, daß er die Wurzel קנמ in bezug auf Jahwe bis zum Augenblick der Zerstörung Jerusalems ebenso gebraucht wie seine Vorgänger, von da ab aber in ganz anderem Sinne. Es ist bekannt, daß er das Verhältnis Jahwes zu seinem Volke wie Hosea und Jeremia unter dem Bilde der Ehe darstellt, dies Bild dabei aber aller seiner Zartheit beraubt. In diesem Zusammenhang gebraucht er denn auch den Ausdruck von der nach ihm in dem fürchterlichen Strafgericht aus, das Jahwe nach c. I6 und 23 über das ehebrecherische Juda bringt. Die alte Beziehung der 
הִִ̣ ist hier deutlich genug beibehalten und ebenso in dem immer noch

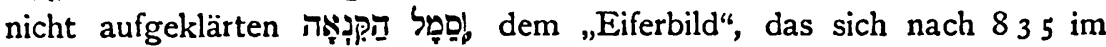
Tempel zu Jerusalem befand. Welchen fremden Gott dies Bild auch dargestellt haben mag, 'was wir vermutlich nie ergründen werden, soviel ist doch klar, daß es eben ein Gottesbild war, durch das nach Hesekiel Jahwes Eifersucht im höchsten Maße wachgerufen wurde, zumal es sich in seinem eigenen Hause befand. Auch in $5 \times 3$ ist die Beziehung der

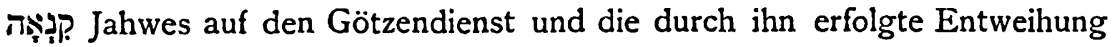
des Heiligtums Jahwes evident.

Soweit stimmt also Hesekiels Sprachgebrauch ganz mit dem vorexilischen überein. Nun aber erfolgt eine scharfe Wendung, und zwar mit dem Augenblicke, in dem durch den Fall Jerusalems und die Zerstörung des Tempels das Strafgericht über Juda vollendet ist. In allen Stücken, die aus späterer Zeit stammen, hat bei Hesekiel die völlig veränderte Beziehung. Sie wendet sich nun nicht mehr gegen das ungetreue Israel-Juda, sondern gegen dessen Feinde. In c. 36 ist es "der

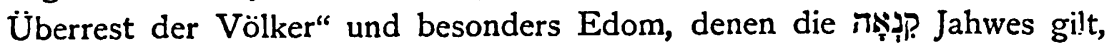
ohne daß aber die Erinnerung an den ursprünglichen Sinn des Ausdrucks schon völlig verloren wäre. Die pִִ̣ Jahwes wird nämlich deshalb rege, weil das Land Kanaan die Schmach der Völker hat tragen müssen, indem es von ihnen erobert und vergewaltigt wurde, während es doch Jahwe gehörte. Es scheint demnach, daß hier noch ein Nachklang von Hoseas ursprünglichem Gedanken vorliegt, nach dem eigentlich das Land Palästina Jahwes Eheweib war, während das Volk Israel durch die Kinder repräsentiert wurde, die dieser Ehe entstammen. Nun ist das Land, also die Gattin, von Fremden mißhandelt; da erwacht die Liebe Jahwes zu seinem Weibe wieder und sie tritt in die.Erscheinung als die sich an ihm vergangen haben. Ebenso steht es in c. 38: in dem Augenblicke, wo Gog mit seinen Scharen Jahwes. Land betritt, lodert Jahwes Grimm und pִ pִ auf und erregt ein gewaltiges Erdbeben, das, mit allerlei furchtbaren Naturerscheinungen verbunden, Gog den Garaus macht in dem Lande, an das er freventlich sich gewagt hat. Damit geschieht zugleich der Liebe Jahwes zu seinem Volke und seiner eigenen Ehre genug, indem dadurch die Völker von ferneren. Angriffen auf das von Jahwe geliebte Land und Volk abgeschreckt und zur Anerkennung von Jahwes übergewaltiger Macht gezwungen werden. So wird am Schlusse des Gog-Abschnittes denn auch in c. 39 dessen Sinn zusammengefabt; und auch hier tritt der Gedanke der hervor, nun aber zusammengestellt mit Jahwes Erbarmen gegen sein Volk Zeitschrift f. d. alstest. Wiss. Jahrg. 28. 1908. 
und bezogen auf Jahwes heiligen Namen, dem er durch sein Eintreten für Israel zu seinem Rechte verhilft.

Durch seine Einfügung in diesen eschatologisch-apokalyptischen Vorstellungskomplex hat nun auch der Gedanke von der הִ Jahwes eine eschatologische Färbung bekommen, die ihm von nun an ziemlich konstant anhaftet. Nur wenige mit Hesekiel ungefähr gleichzeitige oder

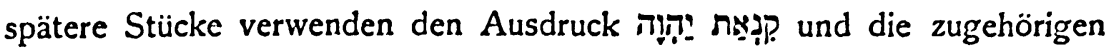
Verbalformen und Adjektiva ohne Beziehung auf die Betätigung Gottes zur Herbeiführung der messianischen Zeit. Es sind dies Num 25 Ir, Ps 7858 und Ps 79 5. Die erstgenannte Stelle gehört der Pinchaserzählung des PC an, in der berichtet wird, wie Pinchas dadurch, dab er die ?ִּק Jahwes ausübte, als die Israeliten sich durch den mit Götzendienst verbundenen Umgang mit heidnischen Weibern versündigten, die drohende

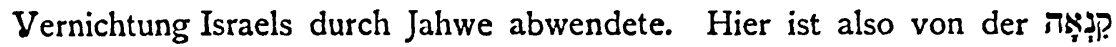
Jahwes in ganz demselben Sinne die Rede, in dem .der Ausdruck in der Zeit vor Hesekiel allein gebraucht wurde. Damit ist aber nicht gesagt, daß man diesem Teil von PC ein über Hesekiel hinaufreichendes Alter vindizieren müsse. Denn in den beiden sicher nachexilischen Psalmen 78 und 79 wird die Wurzel sip in bezug auf Jahwes Verhalten gegen Israel gerade so verwendet. Man hat diesen späten Sprachgebrauch eben auf bewußte oder unbewußte Nachahmung des früheren zurückzuführen.

Alle anderen Stellen, in denen sonst nach Hesekiel die Wurzel mit bezug auf Gott gebraucht wird, verwenden sie in dem Sinne, den sie in der späteren Zeit von Hesekiels Wirksamkeit angenommen hat. So

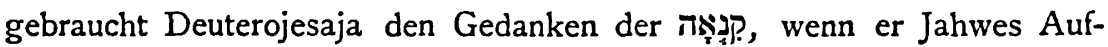
treten zur Herbeiführung der Heilszeit schildert, in 42 13. Da wird Jahwe mit einem heldenhaften Krieger verglichen, der mit lautem Schlachtruf sich selbst anfeuert zur Vernichtung seiner Feinde, an denen er seine Tִ betätigt, indem er sie überwältigt. In den Schlu@kapiteln des Buches Jesaja kehrt der Vergleich Jahwes, der sich zur Aufrichtung seiner Herrschaft anschickt, mit einem Kriegshelden wieder (59 I5 ff.), und in der Schilderung seiner Rüstung wird als Kriegsmantel seine die sich auch hier wieder gegen die Feinde des wahren Israel richtet, die von Jahwe nun den Lohn ihrer Taten empfangen. Sehnsüchtig schaut der Verf. von 6315 nach dem Tage aus, wo Jahwes scheinung tritt, um seinem elenden und gedrückten Volke aus seinen Nöten zu helfen. - Aber auch im ersten Teile des Buches Jesaja finden sich mehrere Stellen, an denen von der ה̦ Jahwes in eschatologischem Sinne die Rede ist, vor allem, wie nicht anders zu erivarten, 'in der 
kleinen Apokalypse c. 24-27; hier wird in 26 Ir die הִָ̣ Jahwes sogar direkt als eine "für sein Volk" wirkende bezeichnet, durch deren Anblick allein die Feinde Israels zu Schanden gemacht werden. In Beziehung zur Aufrichtung des messianischen Reiches steht die 96, was mir neben anderen Gründen doch sehr stark gegen jesajanische Herkunft dieses Stückes zu sprechen scheint. Denn für Jesaja wäre, wie schon MARTI richtig bemerkt, der Gedanke an die Grund zur Freude und Zuversicht, sondern zur Furcht und Besorgnis gewesen. Dasselbe Urteil gilt auch für $3732=$ II Reg 1931, nur daß für diese Stelle die Herkunft von Jesaja schon durch ihre ganze Umgebung, die zweifellos späte Arbeit ist, noch weit sicherer ausgeschlossen wird. Rein eschatologisch ist weiter der Zusammenhang, in dem die ?়ִּ Jahwes bei Sacharja I 14 f. und $82 \mathrm{f}$. erwähnt wird; es handelt sich in beiden Stellen um das endzeitliche Gericht über die Völkerwelt und die herrliche Aufrichtung der Gottesherrschaft in Zion. Jahwes הִ̦ wirkt hier zugunsten Jerusalems und gegen die Heiden, und ist mit Jahwes Grimm und Zorn nahezu synonym. Im Buche Joel 218 begegnen wir noch einmal der schon bei Hesekiel bemerkten Verbindung der Jahwes mit dem Lande; sie regt sich auf die flehentliche Vorstellung des bedrängten Volkes hin, daß durch die Herrschaft der Heiden über Jahwes Land und Volk dieses der Schande preisgegeben und die Macht seines Gottes in Frage gestellt werde, und sie bedeutet dasselbe wie Jahwes Einschreiten zugunsten seines Volkes; dies Einschreiten richtet sich in Joels speziellem Falle auf die Beseitigung der durch eine den Tag Jahwes ankündigende Heuschreckenplage hervorgerufenen Hungersnot. In dieser Heuschreckenplage wird man aber wohl eine symbolische Darstellung des Ansturms der feindlichen Weltmächte zu erblicken haben, der dem Unternehmen Gogs bei. Hesekiel entspricht; 220 und besonders das in c. 4 in Aussicht genommene Völkergericht im Tale Josafat sprechen wenigstens

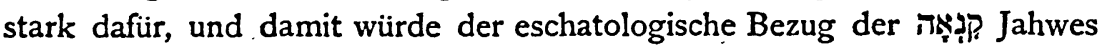
auch bei Joel noch deutlicher hervortreten. Stark eschatologisch ist auch der Zusammenhang, in dem die pִ Tahwes im Buche Zephanja auftritt, so stark eschatologisch, daß dies allein zu erheblichen $Z_{w e i f e l n}$ an der Autheritie der betreffenden Stellen führen mub. Es erscheint mir wenigstens höchst unwahrscheinlich, daß ein Schüler Jesajas, der sich sonst ungefähr in denselben Gedanken wie sein größerer Meister bewegte, ein Gericht über die Bewohner der ganzen Erde einschlieblich der Säugetiere, Vögel und Fische sollte in Aussicht genommen haben. Vielmehr werden die Verse 2 und 3, sowie 17 und 18 des 1 . Kapitels, in denen dieser $4^{*}$ 
Gedanke ausgeführt wird, einem anderen Autor angehören, vielleicht demselben, der im 2. Kapitel seine Drohungen gegen die Juda umgebenden Völker laut werden läht; auch $38 \mathrm{ff}$. könnte man wohl demselben Verfasser zuschreiben, der jedenfalls einer weit späteren Zeit angehören muß als der echte Zephanja. Daß auch Nahum I 2, wo ebenfalls von einer קִנָָאה Jahwes die Rede ist, einem späten eschatologischen Zusammenhang angehört, hat GUNKEL, wie mir scheint, durchaus überzeugend nachgewiesen. Hier wie in den beiden Zephanjastellen ist nun auch jede Beziehung der קִּ̣ Jahwes auf Israels Götzendienst oder auf die Vergewaltigung des gottgeliebten Volkes durch die Heiden geschwunden, -und es steht so, daß einfach da, wo von den "letzten Dingen", vom Weltgericht und von der Aufrichtung des Gottesreichs auf der Erde die Rede ist, unwillkürlich der Ausdruck gänzlich vergessen, was mit diesem Terminus ursprünglich gemeint war, und sucht nur noch nach Worten, die geeignet sind die Gewißheit, dal Jahwe die eschatologischen Wünsche Israels erfüllen werde, recht energisch auszudrücken.

Damit haben wir unseren Gang durch die Geschichte des Gedankens von der Jah Jahwes vollendet und gesehen, wie er aus einem ursprünglich für Israel höchst bedrohlichen allmählich zu einem Heil verhei@enden geworden ist, der schließlich auch noch fast jede Beziehung auf das Volk Israel allein verloren hat und in eine universalistische Gedankengruppe eingetreten ist. Ganz ist er aber doch nicht von dem Geschick des jüdischen Volkes losgelöst worden: denn auch das Weltgericht hat für das Judentum schließlich nur den Sinn, daß in ihm sich seine Herrschaft über die Welt vollende. So endet der Gedanke vom Eifer Jahwes, der ursprünglich Ansprüche Jahwes an sein Volk stark und knapp zusammenfaßte, damit, daß er zum Inbegriff der Ansprüche Israels an die Welt und an seinen Gott wurde und es zum Ausdruck brachte, daß wenigstens einem Teil des Judentums Gott nicht mehr das Ziel ist, nach dem der Mensch sich sehnt und streckt, sondern ein Mittel, wenn auch das höchste, zur Erreichung von irdischen Zwecken.

[Abgeschlossen am 15. November 1907.] 